\title{
Confianza en el Instituto Nacional Electoral mexicano: una perspectiva comparada
}

\section{Confidence in the mexican national electoral institute: A comparative study}

\section{Germán Pérez-Verduzco iD}

Universidad de Colima

german_perez@ucol.mx

\begin{abstract}
Resumen
En México, es claro el enfrentamiento entre el Instituto Nacional Electoral (INE) y Andrés Manuel López Obrador. Con este contexto, es pertinente estudiar el grado de confianza ciudadana en la autoridad electoral y ponerla en perspectiva comparada en los aspectos temporal, institucional y geográfico. Este estudio propone conocer cómo ha evolucionado la confianza en el INE en los últimos años, si en esta institución se confía más o menos que en otras, y qué tanto varía en comparación con otros países latinoamericanos. Dichos aspectos se analizaron mediante estadistica descriptiva y multivariante (HJ-Biplot y Análisis Clúster) con datos del Latinobarómetro 2015, 2016, 2017 y 2018. Los resultados muestran que la confianza de los mexicanos hacia la autoridad electoral ha disminuido a pesar de confiar más en ella que en el gobierno, congreso o los partidos políticos, y que en América Latina la confianza hacia estas tres instituciones está bastante vinculada.
\end{abstract}

Palabras clave: Confianza institucional, confianza politica, Instituto Nacional Electoral (INE), México.

\begin{abstract}
In Mexico, there is an evident confrontation between the National Electoral Institute and López Obrador. In this context, it is important to study citizen confidence in the electoral institution and compare temporary, inter-institutional, and geographically, how has evolved the confidence in the electoral institution in recent years, whether citizens trust it more or less than they do in other institutions, and how it varies compared to other Latin American countries. These aspects were analyzed using descriptive and multivariate statistics (HJ-Biplot and Cluster Analysis) with Latinobarometer's data from 2015, 2016, 2017, and 2018. The results show, on the one hand, that although confidence in Mexican electoral institution was greater than confidence in government, congress or political parties, it has decreased in recent years, and on the other hand, that confidence in the last three mentioned institutions is closely related.
\end{abstract}

Keywords: Institutional confidence, political confidence, National Electoral Institute, Mexico.

Articulo: Recibido el 28 de abril de 2020 y aprobado el 19 de junio de 2020

Cómo citar este artículo:

Pérez-Verduzco, G. (2020). Confianza en el Instituto Nacional Electoral mexicano: una perspectiva comparada. Reflexión política 22(45), pp. 103-115. doi: https://doi.org/10.29375/01240781.3903

\section{Introducción}

En las democracias contemporáneas, la confianza ciudadana en las instituciones es primordial para su funcionamiento y legitimidad. La confianza hacia la autoridad electoral es particular en ese sentido, debido a que esta institución no solo forma parte del juego democrático, sino que a su vez es la encargada 
de administrarlo y verificar que sus reglas se cumplan a cabalidad. En México, el Instituto Nacional Electoral (INE) es la autoridad encargada de organizar no solo los procesos electorales federales, sino que a su vez coordina a los organismos electorales locales para organizar los comicios en las entidades federativas, una capacidad derivada de la reforma constitucional de 2014, la cual además de convertir al IFE (Instituto Federal Electoral) en INE, permitió homologar los estándares organizativos electorales en procesos locales y federales, mejorando con ello la calidad de la democracia en el país.

Este modelo de centralización electoral implicó la transformación de los Institutos Electorales Locales en Organismos Públicos Locales (OPLES), cambio que quitó influencia a los gobernadores estatales en el sentido de que ahora el INE sería el encargado de designar a los integrantes de las OPLES (Ayala y Triana, 2020). La organización de los comicios locales estaba tan viciada que los gobernadores prácticamente controlaban las instituciones electorales estatales (Torres-Ruiz, 2014), situación que atentaba contra la democracia subnacional. Esta clase de prácticas continúan enraizadas en varios liderazgos políticos mexicanos por su incapacidad para abstraerse de los más de 70 años de un contexto socio-histórico y político teñido de nepotismo, corrupción, autoritarismo y represión impuestos por un gobierno de partido hegemónico.

En la primera mitad del siglo XX, los comicios mexicanos representaban un peligro latente para la ciudadanía debido a los violentos enfrentamientos por el control de las casillas para votar, ya que entonces la ley electoral planteaba que los primeros electores en llegar al lugar serían los encargados de dirigir las elecciones ahí, lo que llevaba a una importante cantidad de muertes (Corona, 2002). En la segunda mitad del siglo, periodo en que el escritor Vargas Llosa calificó al país como la "dictadura perfecta”, las elecciones se caracterizaron por el fraude. Primero, por su condición de ejercicios meramente simbólicos y después por las "fallas" del sistema. Empero, en ambos casos la competencia electoral era básicamente simulada.

Aunque en la literatura no hay consenso al respecto, aquí se coincide con el punto de vista de que los avances fundacionales en el país en materia de democracia política comenzaron en el ámbito federal a partir de las reformas electorales realizadas entre 1990 y 1996, donde a través de la fundación de un órgano independiente del gobierno para la organización de los comicios se pudieron sentar las bases para la realización de elecciones competitivas, lo que dio paso del dominio de un solo partido al pluripartidismo y de la manipulación electoral a la transparencia (Méndez y Loza, 2013). Desde las primeras elecciones organizadas por el IFE (1991), existió cierta desconfianza hacia el organismo, motivo por el cual en los años posteriores fue necesario llevar a cabo varias reformas enfocadas a la conformación de las mesas directivas de casillas y así dotar de mayor credibilidad a estos procesos (Woldenberg, 2010).

En concreto, en el periodo 1990-1996 se hicieron cuatro importantes reformas electorales: I) la creación del IFE (1990), que implicó el surgimiento de una institución imparcial para brindar certeza y legalidad a las elecciones; II) la reforma al Código Federal de Instituciones y Procedimientos Electorales (1993), que daba al IFE la atribución de dar validez y constancia a las elecciones de diputados y senadores, además de establecer límites respecto a los gastos de campaña; III) la instauración de la figura de los Consejeros Ciudadanos (1994), con la cual los partidos políticos quedaban con solo un representante (con voz pero sin voto) en el Consejo General; y IV) la modificación al artículo 41 constitucional (1996), que aumentaba la independencia y autonomía del IFE respecto al Poder Ejecutivo y brindaba mayor peso en su integración al Poder Legislativo y la ciudadanía.

Si bien es cierto que el IFE ha estado involucrado en varias controversias desde su fundación, sobre todo por algunos conflictos postelectorales que le provocaron descalificaciones procedentes de diversas fuerzas políticas del país, también es verdad que su creación contribuyó a tener un México con mayor pluralidad, equidad y justicia electoral, así como partidos políticos más fuertes y competitivos (Torres-Ruiz, 2014). Prueba de ello son las elecciones intermedias de 1997 y las presidenciales del 2000, las cuales sin duda constituyeron un punto de quiebre para la democracia mexicana. Incluso hay evidencia de que en los años posteriores a la alternancia del 2000 se dio un notable incremento en la confianza política (Moreno, 2003; Temkin, Salazar y Ramírez, 2004), lo que indirectamente dotaba de legitimidad al trabajo realizado por el IFE. 
La que quizás pudiera considerarse como la primera gran crisis del instituto, son los resultados electorales de 2006, en los que el candidato del Partido Acción Nacional (PAN), Felipe Calderón Hinojosa, fue elegido como presidente. Estas elecciones no solo han sido las más competidas de la historia (con una diferencia de solo medio punto porcentual entre el primer y segundo lugar), sino también las más cuestionadas debido a varias irregularidades durante el proceso y a que la jornada electoral estuvo llena de incidentes (Valles, 2016). De hecho, la inconformidad del entonces candidato del Partido de la Revolución Democrática (PRD), Andrés Manuel López Obrador (AMLO), fue tal que, después de varias protestas y bloqueos en la capital del país, algunos meses posteriores a su derrota electoral se autoproclamó "presidente legítimo" de México.

Cabe mencionar que en los años posteriores a esos comicios, los consejeros del IFE manifestaron cierta proximidad a diversos partidos políticos, situación que comenzó a restarle legitimidad. Sin embargo, el proceso electoral de 2012 fue menos complejo para el instituto gracias a una amplia ventaja del entonces candidato Enrique Peña Nieto sobre AMLO, quien era su más cercano perseguidor. Durante este sexenio se hicieron diversas modificaciones constitucionales, entre ellas la reforma estructural en materia electoral. En esencia, la reforma de 2014 buscaba desarrollar la democracia en las entidades federativas del país (Ayala y Triana, 2020).

Una de las mejoras es el fortalecimiento de las condiciones de equidad en la contienda electoral, pues la resolución de procedimientos sancionadores expeditos ya no recae en el INE sino en el Tribunal Electoral del Poder Judicial de la Federación (TEPJF). Tal delimitación hace posible que el INE pueda concentrarse en la organización de procesos electorales, dejando las cuestiones jurídicas al TEPJF, que entre sus facultades cuenta con la posibilidad de anular elecciones si: a) se excede el gasto de campaña autorizado en un cinco por ciento; b) se compra tiempo o cobertura informativa en radio y televisión; o c) se reciben o utilizan recursos ilícitos o públicos ${ }^{1}$. Finalmente, la reforma constitucional también establece obligaciones al INE en cuanto a la vigilancia de los recursos otorgados a partidos políticos, siendo su atribución fiscalizarlos tanto a nivel federal como local, lo que sin duda suma transparencia.

En la actualidad, el Consejo General del INE lo conforman 11 ciudadanos elegidos por la Cámara de Diputados: diez Consejeros Electorales y un Consejero Presidente. Además, la institución tiene un Servicio Profesional Electoral Nacional (SPEN) para garantizar la imparcialidad y capacidad técnica de los funcionarios que se involucran en la organización de elecciones federales y locales. Otras funciones que puede realizar el INE es organizar elecciones de los dirigentes de partidos políticos (si estos lo solicitan), vigilar que los candidatos independientes puedan acceder a ciertos tiempos del Estado en los medios de comunicación tradicional (radio y televisión), verificar que se cumpla el $2 \%$ (mínimo requerido) de la lista nominal cuando se soliciten consultas populares y, en caso de llevarse a cabo, organizarlas y difundir sus resultados. Este aumento de atribuciones está en consonancia con la evolución global de los sistemas electorales, pues los organismos electorales ya son responsables de tareas tan diversas como la emisión de documentos de identificación del elector, el trazado de límites geográficos de los distritos o áreas electorales y la aplicación de mecanismos de democracia directa (García, 2016).

A pesar de todos los cambios derivados de la reforma constitucional y de que el desarrollo del INE apunta hacia una mejor democracia electoral en México, hay estudios que reportan que la reforma no ha tenido un impacto estadísticamente significativo en la calidad de la democracia a nivel subnacional, medida a través de las dimensiones de calidad de la ciudadanía, desempeño de la autoridad electoral y el sistema de partidos (Ayala y Triana, 2020). Además, en el plano local aún hay acciones que impactan en las tres dimensiones operativas de los procesos electorales: el financiamiento y gasto de campaña, las capacidades de fiscalización y acreditación de gastos electorales y el modelo de comunicación política; aspectos que al conjugarse provocan contiendas electorales desiguales (Alarcón, 2016). Por tanto, es evidente que el instituto tiene tareas pendientes en cuanto a la mejora de su desempeño como autoridad electoral.

${ }^{1}$ Cabe señalar que cualquiera de las violaciones tendría que acreditarse de forma objetiva, siendo estas determinantes cuando la diferencia entre los votos conseguidos por el primer y segundo lugar sea menor al cinco por ciento. 
En ese sentido, uno de los principales desafíos actuales para el instituto son sus diferencias con AMLO, quien a lo largo de su trayectoria política y en reiteradas ocasiones ha cuestionado el actuar del organismo. Paradójicamente, es justo a partir de su victoria en las elecciones de 2018 cuando el INE parecía recuperar buena parte de su credibilidad. Sin embargo, las fricciones entre el líder del ejecutivo $\mathrm{y}$ el instituto han aumentado en últimas fechas por la próxima renovación del Consejo General y el nombramiento de cuatro consejeros electorales que entrarian en funciones este año, apenas unos meses antes de las elecciones intermedias de 2021. Con dicho contexto, y considerando que el actual presidente de la república goza de los más elevados niveles de popularidad en la historia, es pertinente investigar cómo se encuentra la confianza ciudadana en el INE, ya que eso permitiría tener una noción sobre la fortaleza o debilidad del instituto en cuanto a la opinión pública y ante posibles embates del gobierno $\mathrm{u}$ otros actores políticos afines.

Al hablar de confianza en el ámbito sociopolítico, se hace referencia a la esperanza ciudadana en que los individuos que los representan en el espacio público, y que por tanto tienen más poder, actuarán buscando el bien común (Valdez y Huerta, 2018). Dependiendo de si tal expectativa se pone en personas o instituciones se le denomina confianza social, en el primer caso, o confianza política, en el segundo (Montero, Zmerli y Newton, 2008; Palazuelos, 2012). La confianza política se refiere a las valoraciones ciudadanas sobre las instituciones centrales del sistema político (Denters, Gabriel y Torcal, 2007), y a la creencia de que estas actuarán siempre de manera justa, igualitaria y correcta, evitando aquellas acciones perjudiciales para el país (Montero et al., 2008). En otras palabras, qué tanto esperan los ciudadanos que las instituciones actúen en favor del bien común o con la idea de alcanzar el beneficio público.

La confianza ciudadana no se establece de un día para otro, sino que es una construcción (Woldenberg, 2010). Landau (2009) establece que la confianza es de índole relacional, y que esta supone una relación con el saber (solo se conoce una parte de la información puesto que se está en medio del saber y el ignorar); con la autoridad (a la que se reconoce y sobre la que se acepta cierta dependencia); con el futuro (lo que se espera que ocurra); y, con el pasado (lo que antes ha sucedido). Aunque, de igual manera, podría decirse que en realidad se trata solo de tres aspectos: incertidumbre sobre lo que acontecerá (información incompleta), una relación de dependencia hacia cierta autoridad, y la evaluación de las experiencias previas. Como apunta Palazuelos (2012), este último aspecto de evaluación retrospectiva es la clave para entender la confianza política, al menos desde el enfoque del desempeño gubernamental, teoría que plantea que la eficacia de los gobiernos en diversos aspectos influye directamente sobre el nivel de confianza ciudadana en las instituciones políticas (Segovia, Haye, González, Manzi y Carvacho, 2008).

Las acciones gubernamentales en problemáticas sociales como la pobreza, el desempleo, la inseguridad o la corrupción, son esenciales para la generación de confianza debido a que "los ciudadanos tenderán a confiar más en las instituciones políticas cuando las perciban como capaces para llevar a cabo sus respectiva funciones" (Palazuelos, 2012, p. 87). En su defecto, dichas problemáticas estimulan la desconfianza y la pérdida de legitimidad de las autoridades políticas, haciendo crecer con ello el poder de los populismos o autoritarismos que atentan contra la estabilidad democrática (Inglehart y Norris, 2017).

Por otra parte, aunque las experiencias de la ciudadanía con las instituciones o autoridades que las representan no son un elemento constitutivo de la confianza, sí lo es la evaluación sobre su desempeño o desenvolvimiento. Es decir que la confianza institucional siempre está en función de la efectividad percibida en las acciones de la institución evaluada (Segovia et al., 2008). De ahí que se haya encontrado que los ciudadanos que confian más en sus instituciones residen en lugares en los que en general se percibe mayor calidad en los servicios públicos (Monsiváis, 2019). Esto no debería sorprender porque la percepción del desempeño gubernamental está claramente ligada a la capacidad de respuesta y resolución del sistema político para aspectos elementales como el mantenimiento del orden o la producción de riqueza (Moreno, 2003).

Con todo, la evaluación que la ciudadanía hace sobre las instituciones es influida por aspectos tan racionales como la estimación de los posibles riesgos, pérdidas o ganancias al confiar en ellas (evaluación costo-beneficio), o por otros más emocionales como la calidad de la interacción, es decir, si recibió un trato positivo o negativo. Con relación a ello, existe evidencia de que el 
impacto de las experiencias ciudadanas negativas es mucho mayor al de las positivas cuando se evalúan servicios públicos (Kampen, Van de Walle y Bouckaert, 2006). Esto lleva a suponer que para subsanar la desconfianza política se requiere un esfuerzo mayúsculo de las instituciones para mejorar su desempeño, y que así la ciudadanía pueda valorarlas y confiar en ellas.

Desde hace décadas existe una amplia desconfianza hacia las instituciones de la democracia en América Latina. Entre 1996 y 2017, la confianza en los partidos políticos y congresos nunca llegó siquiera al 40\% (Monsiváis, 2019). En el periodo 1995-2015, el rango de confianza hacia los primeros osciló entre $13 \%$ y $36 \%$, y entre $21 \%$ y $40 \%$ hacia los segundos (Corporación Latinobarómetro, 2015). En cambio, en el mismo lapso de tiempo, las autoridades electorales han sido las instituciones de la democracia que gozan de mayor confianza en la región (García, 2016). En el caso mexicano, la Encuesta Mundial de Valores 2010-2014 señaló que las instituciones con mayor confianza eran las universidades, las organizaciones de mujeres, las medioambientales y la Iglesia, mientras que instituciones de la democracias como el gobierno o los partidos políticos se encontraban en los últimos lugares (Institute for Comparative Survey Research, 2014).

Las investigaciones centradas en analizar específicamente la confianza de la ciudadanía en la autoridad electoral del país son escasas. Se sabe, por ejemplo, a partir de datos de Consulta Mitofsky, que la confianza en el IFE/INE ha descendido gradualmente, pues al revisar las calificaciones otorgadas por la ciudadanía a la institución en las tres últimas elecciones presidenciales se hallaron puntajes de 6.9 en el 2006, 6.7 en el 2012, y 6.5 en el 2018; mientras que en las elecciones intermedias, el puntaje fue 7.2 en el 2009 y 6.1 en el 2015 (Juárez, 2019).

En un estudio realizado por el propio Instituto Nacional Electoral y el Colegio de México (2014), se encontró que el todavía IFE contaba con apenas el 34\% de la confianza ciudadana. A este trabajo pueden añadirse algunas otras investigaciones que a su vez reportan una gran desconfianza hacia la institución electoral mexicana (Barrientos, 2010; García, 2016; Heras, 2011; Ortega y Somuano, 2014; Torrico, 2015), pero a pesar de las valiosas contribuciones realizadas por ellas, estas poseen algunas limitaciones: a) casi todas se realizaron previo a la reforma constitucional de 2014; b) algunas utilizaron datos de las ediciones 2004, 2006, 2008, 2010, 2012 y 2014 del Barómetro de las Américas o de la extinta Encuesta Nacional sobre Cultura Política y Prácticas Ciudadanas (ENCUP) cuyas ediciones 2001, 2003, 2005, 2008 y 2012 refieren la percepción hacia el entonces IFE; c) unas analizaron la confianza hacia la institución pero a nivel local; $y$, d) otras centraron sus análisis en la confianza de los congresistas o parlamentarios en el organismo electoral, más que a estudiar la confianza de la ciudadanía.

Debido a la situación actual en el contexto político mexicano, descrito hace algunos párrafos, y las lagunas identificadas en la literatura al respecto, cabe preguntarse ¿cómo ha evolucionado la confianza en la autoridad electoral desde su transformación de IFE a INE, es decir, después de la reforma constitucional de 2014?, ¿los mexicanos confían en el INE en mayor o menor medida de lo que lo hacen en otras instituciones de la democracia?, ¿qué tanto confían los mexicanos en su autoridad electoral en comparación con los ciudadanos de otros países latinoamericanos? Con la finalidad de responder tales interrogantes, los objetivos de esta investigación fueron: I) analizar los niveles de confianza en el INE correspondientes al periodo de los dos últimos procesos electorales (2015-2018); II) contrastar los niveles de confianza depositada en el INE con los existentes hacia el gobierno, el congreso y los partidos políticos en tal periodo; y III) realizar un análisis comparado entre México y los demás países latinoamericanos en función de la confianza hacia estas cuatro instituciones entre el año 2015 y el 2018.

\section{Método}

El presente trabajo es un estudio comparado y cuantitativo en que se recurrió específicamente a estadísticos descriptivos para los primeros dos objetivos, y a estadística multivariante para el tercero de ellos. Los análisis descriptivos se llevaron a cabo con el software SPSS Statistics (versión 22) y el programa Excel. El análisis multivariante se realizó a través del método HJ-Biplot y un Análisis Clúster, cuyas representaciones gráficas se elaboraron mediante el programa MultBiplot ${ }^{2}$.

${ }^{2}$ Software desarrollado por José Luis Vicente-Villardón, profesor-investigador en la Universidad de Salamanca. 
El HJ-Biplot pertenece a la familia de los métodos Biplot, representaciones gráficas a baja dimensión de una matriz de datos $X$ ( $i$ individuos y $j$ variables). Es decir que los métodos Biplot sirven para interpretar los elementos de una matriz $X_{i x j}$ como productos internos de vectores utilizados para representar sus filas y sus columnas. El HJBiplot en concreto, consiste en una representación gráfica multivariante de las líneas de una matriz $X_{j x h}$ mediante los marcadores $j_{1} \ldots j_{n}$ para sus filas y $h_{1} \ldots . . h_{p}$ para sus columnas, elegidos de forma que ambos marcadores sean superpuestos en un mismo sistema de referencia con máxima calidad de representación y con idéntica bondad de ajuste tanto para las filas como para las columnas (Galindo, 1986).

Al interpretar un HJ-Biplot siempre debe considerarse lo siguiente. Los marcadores fila (individuos) se representan con puntos y los marcadores columna (variables) como vectores. La dirección de los vectores columna refleja la dirección en la que se incrementan los valores de la variable correspondiente, y las proyecciones de todos los puntos fila sobre un vector en particular reproducen aproximadamente la ordenación de los elementos o individuos respecto a dicha variable. La distancia entre los individuos (en este caso, países) indica disimilaridades entre ellos, y la proximidad, su grado de similitud con relación a las distintas variables. Respecto a estas, mientras más longitud tenga un vector mayor es la variabilidad de la variable. El grado de covariación entre variables se refleja en los ángulos formados entre los vectores: mientras más pequeños sean, mayor es la correlación; ángulos llanos muestran relaciones inversas o negativas entre ellas, y ángulos rectos indican independencia entre las mismas.

Los métodos Biplot pueden complementarse bien con otras técnicas de estadística multivariante como el Análisis Clúster, también llamado Análisis de Conglomerados. Con esta técnica se busca reducir la cantidad de información en pequeños grupos o clústeres, donde los miembros comparten características similares (Lin y Chen, 2006); es decir, formar grupos de individuos, objetos, lugares, variables, etc., en función de su similaridad. Las técnicas de agrupamiento pueden ser no jerárquicas o jerárquicas, y estas últimas a su vez ser divisivas o aglomerativas; además, los procedimientos de aglomeración pueden realizarse por encadenamiento simple, completo, por método de Ward o por método del centroide. En este trabajo se implementó un análisis clúster jerárquico aglomerativo por método de Ward que consistió en agrupar los países en función de maximizar la homogeneidad intra clúster.

Los datos analizados se obtuvieron de las encuestas Latinobarómetro de los años 2015, 2016, 2017 y 2018, las cuales abarcan los dos últimos procesos electorales mexicanos: las elecciones intermedias de 2015 y las presidenciales de 2018. Cada una de las bases de datos se encuentra disponible sin costo en el sitio web de la organización ${ }^{3}$, en el apartado de "Banco de Datos". Para llevar a cabo los análisis, fue necesario descargar los cuatro archivos de estas ediciones y elaborar uno nuevo en el que únicamente se incluyeran los reactivos de interés, es decir, aquellos correspondientes a la confianza en el gobierno, en el congreso, en los partidos políticos y en la institución electoral. Tales ítems eran de escala tipo Likert con cuatro opciones de respuesta: mucha confianza, algo de confianza, poca confianza y ninguna confianza. Como en las bases de datos originales el grado de confianza estaba graduado de menos a más (mucha $=1 ;$ algo $=2 ;$ poca $=3$; ninguna $=4$ ), se decidió transformar los valores de respuesta al orden inverso para hacer más clara la interpretación de resultados, de forma que los valores más altos indican mayor confianza institucional.

En general, la muestra de encuestados latinoamericanos en cada uno de los años que se analizaron fue de 20,250 ciudadanos en el 2015; 20,204 en el 2016; 20,201 en el 2017; y 20,204 en el 2018. En cuanto a los datos de encuestados mexicanos, la muestra analizada fue de 1,200 ciudadanos en cada año entre el 2015 y el 2018.

\section{Resultados}

Respecto a los primeros dos objetivos del estudio, los datos apuntan a que la confianza de los mexicanos en el INE ha disminuido en los últimos años (Figura 1). Además, es la única de las instituciones analizadas que no tuvo un aumento de confianza el año posterior a las elecciones intermedias de 2015. Esta situación permite establecer que la institución electoral mexicana

${ }^{3}$ http://www.latinobarometro.org/lat.jsp 
presentó una notable tendencia negativa respecto al nivel de confianza ciudadana entre los años 2015 y 2017, y que esta inercia se detuvo en el 2018.

Figura 1. Confianza hacia las instituciones de la democracia en México (2015-2018).

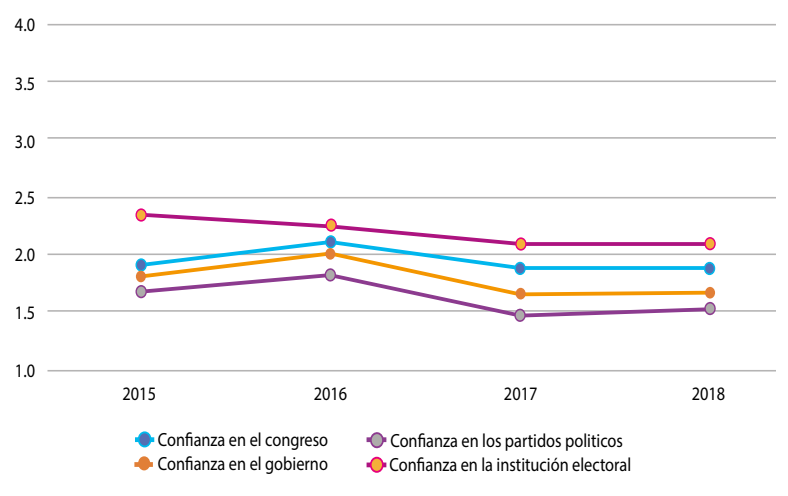

Fuente: Elaboración propia a partir de datos del Latinobarómetro 2015, 2016, 2017 y 2018.

Sobre las otras instituciones de la democracia, puede decirse que la disminución de confianza no ha sido constante. Al no existir por lo menos dos claros decrementos consecutivos, sería inexacto hablar de una tendencia negativa en cuanto a los niveles de confianza institucional. Por ejemplo, la confianza en los partidos políticos aumentó de 2015 a 2016, disminuyó de 2016 a 2017, y de nuevo aumentó un poco en el 2018. Lo mismo ocurre con el gobierno y el congreso, aunque en el caso de estos ni siquiera se dio ese pequeño aumento de confianza el último año; si acaso mantuvieron los niveles de 2017. Lo que sí puede afirmarse, en cambio, es que la confianza de la ciudadanía hacia estas tres instituciones no ha alcanzado los niveles del 2016.

La figura anterior muestra que a pesar de que la confianza hacia el INE ha venido decreciendo, esta institución es, de las cuatro incluidas en el estudio, en la que más confian los mexicanos porque todos los años registró promedios más elevados que las otras. Mientras que el INE tuvo medias de confianza de entre 2 y 2,5 durante esos cuatro años, los promedios de las demás instituciones se encontraban entre 1,4 y 2,1. Dicho de otra forma, la confianza hacia la institución electoral ha estado ligeramente por encima de la "poca confianza", y la depositada en el gobierno, el congreso y los partidos políticos se ha encontrado incluso por debajo de esa categoría.

Comparando entre instituciones electorales latinoamericanas, se aprecia que durante el periodo 2015-2018, básicamente todos los países de la región padecieron una constante disminución en la confianza (Figura 2). Al igual que México, Argentina, Ecuador, El Salvador, Panamá, Perú y Uruguay, presentan tendencias negativas. Por su parte, en Brasil, Chile, Colombia, Costa Rica, República Dominicana, Guatemala y Paraguay, existió idéntico comportamiento hasta el 2018, año en que la inercia se rompió y la confianza en la autoridad electoral aumentó de nuevo. Finalmente, en Bolivia, Honduras, Nicaragua y Venezuela, la confianza se ha comportado de forma irregular.

Figura 2. Confianza en las instituciones electorales latinoamericanas (2015-2018).
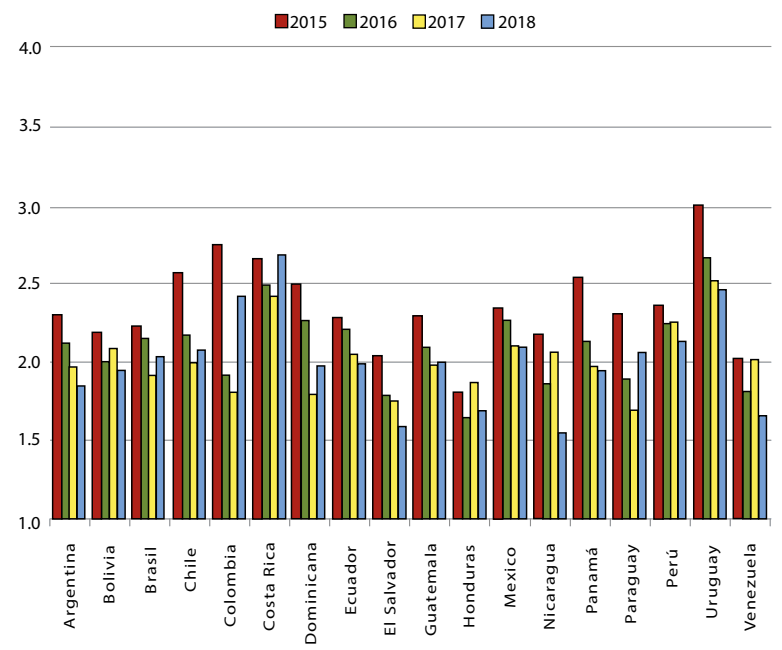

Fuente: Elaboración propia a partir de datos del Latinobarómetro 2015, 2016, 2017 y 2018.

En términos globales, los países con mayor confianza en la institución electoral en el periodo de estudio son Uruguay y Costa Rica. En tercer y cuarto lugar aparecen Perú y Colombia, respectivamente, y en el quinto están empatados México y Chile. Los países con menor confianza en la autoridad electoral son Honduras, El Salvador, Venezuela, Nicaragua y Paraguay, todos con una media de confianza inferior a 2 (Figura 3). 
Figura 3. Promedio de la confianza en las instituciones electorales latinoamericanas (2015-2018).
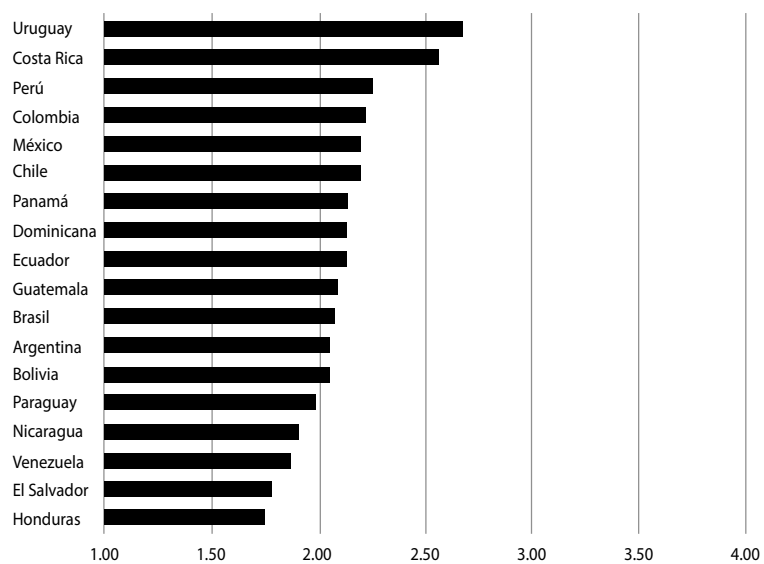

Fuente: Elaboración propia a partir de datos del Latinobarómetro 2015, 2016, 2017 y 2018.
Al comparar los resultados de las cuatro instituciones de los 18 países en tal periodo de tiempo a través del método HJ-Biplot se encuentran cosas interesantes (Figura 4). Primero, hay que mencionar que los dos ejes principales del gráfico acumulan hasta 90\% de la varianza, es decir, que estos explican tal porcentaje de variabilidad de los datos. El primer eje absorbe hasta el 68,7\% de variabilidad, y por tanto, explica la mayor parte de la información. Esto implica que el primer eje es más importante que el segundo para discriminar entre individuos, es decir, que las diferencias más significativas entre los países están en el grado de confianza hacia los partidos políticos, congresos y gobiernos, es decir, en los representantes políticos, más que en la confianza depositada en las instituciones electorales.

Figura 4. HJ-Biplot con datos sobre la confianza hacia las instituciones de la democracia en América Latina (2015-2018).

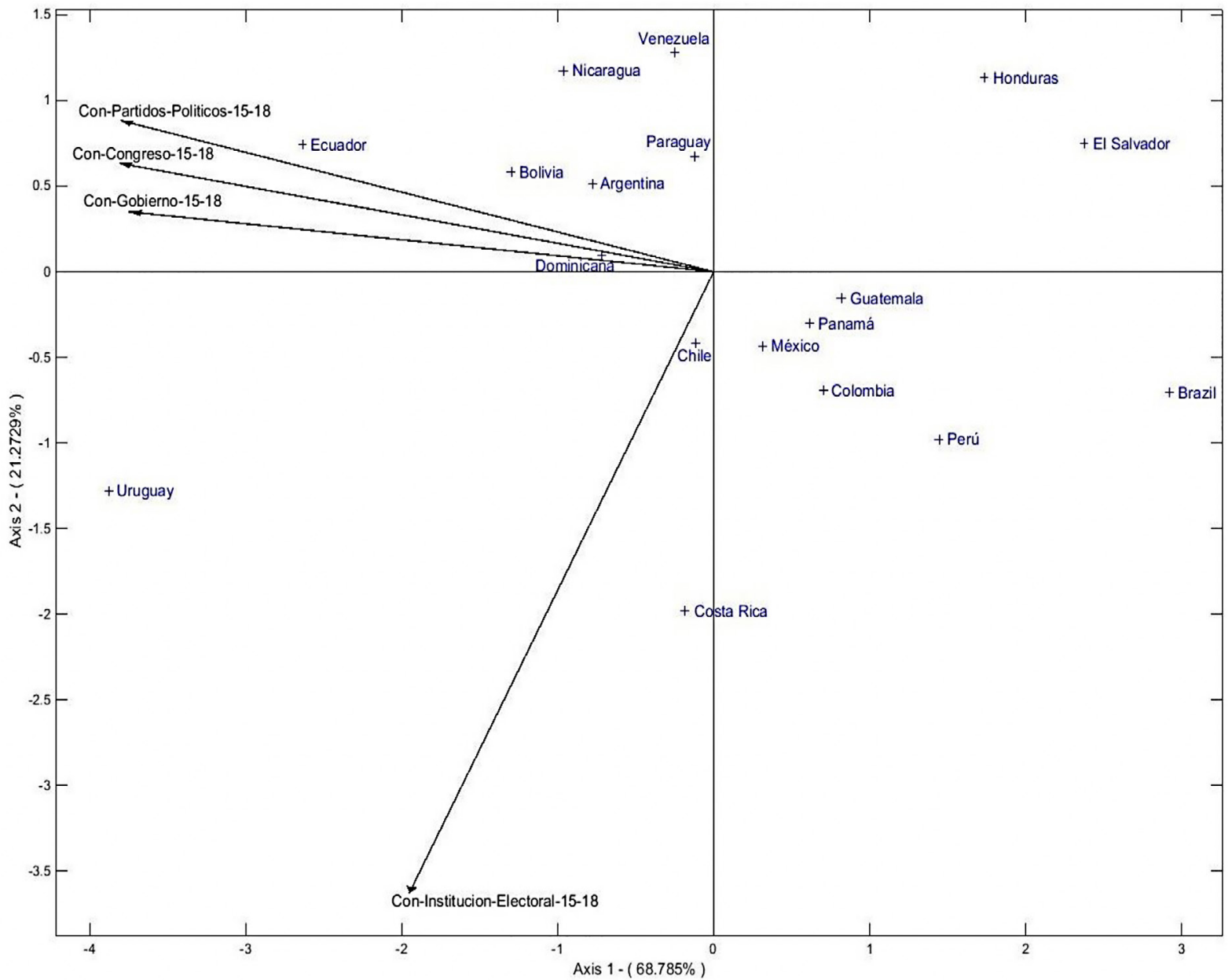

Fuente: Elaboración propia a partir de datos del Latinobarómetro 2015, 2016, 2017 y 2018. 
En cuanto a las variables examinadas, por un lado se aprecia un alto grado de correlación entre los niveles de confianza hacia los partidos políticos, los congresos y los gobiernos; y por el otro, que la confianza hacia las instituciones electorales parece ser independiente a la depositada en las otras instituciones de la democracia. Realizando un análisis sobre la posición de los países en función del primer eje, que constituye el componente principal en el HJ-Biplot, se aprecia que aquellos con mayor confianza en el gobierno, el congreso y los partidos políticos durante este cuatrienio en la región fueron los ciudadanos uruguayos y ecuatorianos. En contraste, quienes menos confiaban en tales instituciones eran los ciudadanos brasileños, hondureños y salvadoreños. Respecto al segundo eje, se observa que Uruguay y Costa Rica fueron los países donde sus ciudadanos han confiado más en la institución electoral en este cuatrienio, mientras que Honduras, El Salvador y Venezuela, fueron aquellos en los que sus ciudadanos tenían menos confianza hacia la misma.

$\mathrm{Al}$ añadir el análisis de clúster jerárquico con método de Ward al HJ-Biplot, se obtiene una representación gráfica aún más ilustrativa, ya que en ella se aprecian cuatros agrupaciones de países creados en función de su similaridad respecto a estas variables (Figura 5).

Figura 5. HJ-Biplot y Análisis de Clústeres (agrupamiento jerárquico con método de Ward) con datos sobre la confianza hacia las instituciones de la democracia en América Latina (2015-2018).

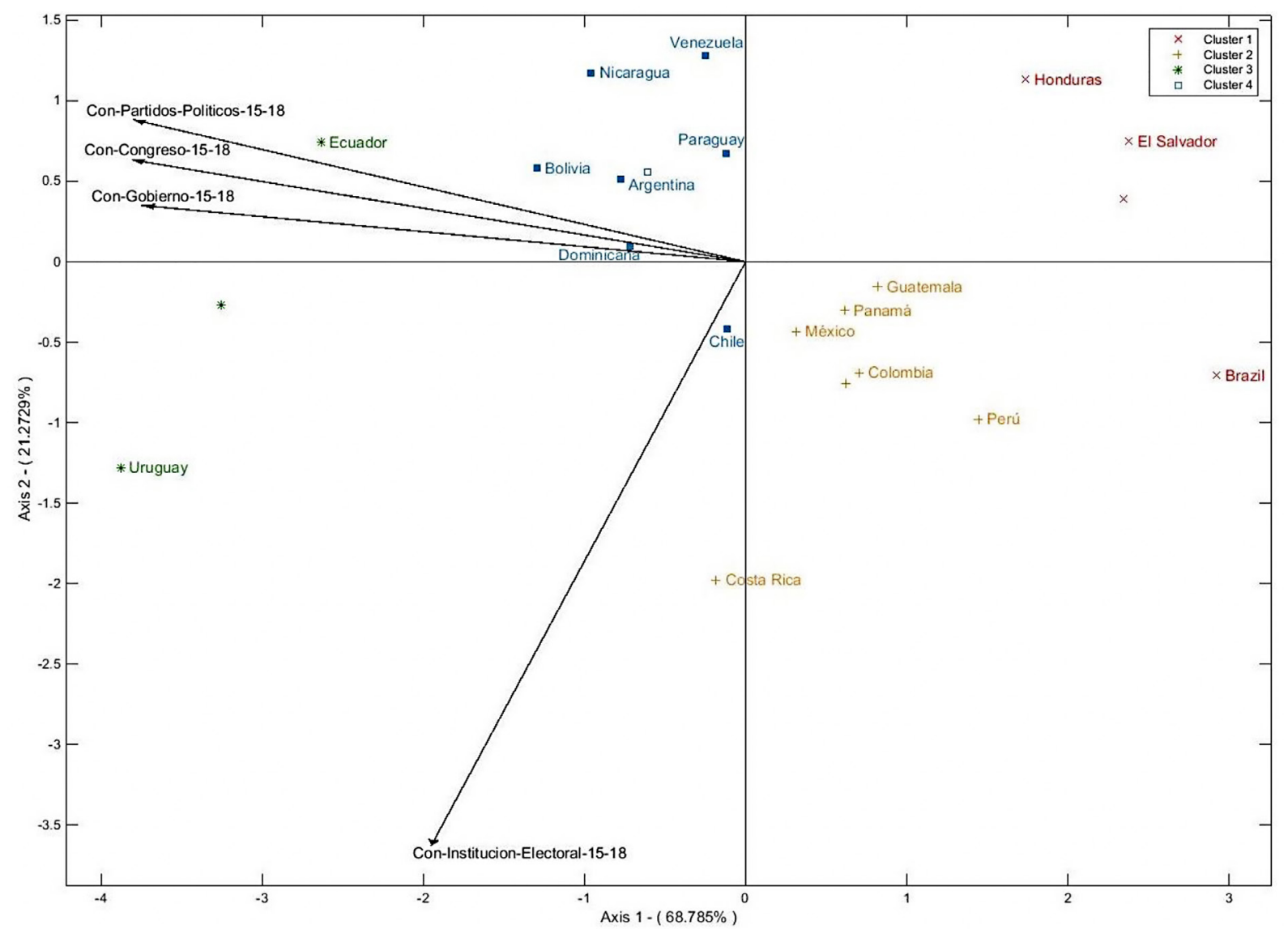

Fuente: Elaboración propia a partir de datos del Latinobarómetro 2015, 2016, 2017 y 2018.

El clúster 1, formado por Brasil, Honduras y El Salvador, sería el que presenta menor confianza política en general, es decir, los peores niveles de confianza en las cuatro instituciones. México estaría en el clúster 2, junto a Panamá, Guatemala, Colombia, Perú y Costa Rica, que podrían considerarse un conjunto de países con cierta confianza en la institución electoral, pero muy poca hacia sus gobiernos, congresos y partidos políticos. Luego, el clúster 3 lo forman República Dominicana, Chile, Argentina, Paraguay, Bolivia, Nicaragua y Venezuela, países que presentaron cierta confianza hacia estas tres últimas instituciones (o al menos más que los países de los clústeres 1 y 2), pero 
menos hacia la institución electoral. Por último, Uruguay y Ecuador serían el clúster 4, es decir, los países con mayor confianza política en la región en el periodo 2015-2018.

\section{Conclusiones}

Los resultados de este trabajo señalan que aunque desde hace tiempo los mexicanos confian en el INE en mayor medida de lo que lo hacen en el gobierno, el congreso y los partidos políticos, esta confianza ha decrecido de manera gradual en los últimos años. Pareciera que ni la reforma constitucional de 2014, ni las alternancias de partidos en el poder derivadas de los dos últimos procesos electorales de 2015 y 2018, han tenido algún efecto en la confianza de los mexicanos hacia la autoridad electoral. Esta disminución de la confianza en el INE se vuelve aún más preocupante si al escenario se añaden las siguientes cuestiones.

Uno de los principales peligros para el instituto son sus diferencias con el presidente de la república. Las constantes críticas y señalamientos de AMLO, quien como ya se ha dicho goza de bastante popularidad entre los mexicanos, seguramente incidirán en la opinión pública, pudiendo convertirse en un factor perjudicial para la imagen del INE, y derivar en un mayor declive de la confianza ciudadana de cara al importante proceso político que representarán las elecciones intermedias de 2021, donde la confianza hacia el organismo sin duda será un aspecto elemental para el fortalecimiento o debilitamiento de la democracia en el país.

Los datos analizados brindan información sobre la percepción ciudadana existente en los últimos cuatro años previos a la alternancia en el poder. Si bien es cierto que entre 2015 y 2018 la confianza hacia el INE era mayor que la depositada en otras instituciones de la democracia, eso ocurría cuando el gobierno era priista y el líder del ejecutivo era Peña Nieto, quien dejó el cargo como el presidente con la aprobación más baja en los últimos cinco sexenios. La situación puede cambiar cuando la ciudadanía tenga que posicionarse entre apoyar al popular y carismático presidente actual, o a la frecuentemente cuestionada y más distante autoridad electoral.

Además, aunque podría plantearse (y con razón) que en un régimen democrático la opinión pública no debe determinar las decisiones del
Estado, y que por mucho que aquella influya, estas en realidad dependen de las leyes e instituciones que lo respaldan, es indudable que la situación no luce favorable para el INE, pues del análisis no puede omitirse el hecho de que los cuatro nuevos consejeros de la autoridad electoral mexicana serán nombrados por un congreso conformado en su mayoría por diputados de MORENA (Movimiento de Regeneración Nacional), partido político del que proviene el presidente.

Por otro lado, desde el punto de vista comparado puede concluirse que a pesar de lo acontecido en el contexto político mexicano, durante el cuatrienio analizado el INE es una de las instituciones electorales con mayor confianza ciudadana en América Latina, estando solo por debajo de los organismos equivalentes en Uruguay, Costa Rica, Perú y Colombia. El enfoque comparativo dejó ver que entre 2015 y 2018, México formó parte de un conjunto de países (al que también pertenecían Perú, Panamá, Guatemala, Colombia y Costa Rica) con poca confianza política.

Lo más interesante de este grupo es que, aunque tres de los países tuvieron elecciones presidenciales en 2018 (México, Costa Rica y Colombia), no se aprecia ningún patrón en sus contextos políticos ni en sus comportamientos electorales con relación a la confianza. Mientras que en México la confianza hacia la institución electoral disminuyó un poco, en Colombia y Costa Rica se registró un incremento. Esto evidencia que la alternancia en el poder no siempre se traduce en mayor confianza institucional, pues a pesar de que tanto en Colombia como en México se presentó la primera, la segunda solo aumentó en Colombia. De igual modo, aunque en Costa Rica no se dio una alternancia en el poder y en México sí la hubo, solamente en Costa Rica incrementó la confianza hacia la institución electoral.

Honduras, El Salvador y Brasil fueron los países de la región con los niveles más bajos de confianza durante este periodo, y no solo respecto a la institución electoral sino también en cuanto a la confianza política en general. En los dos últimos, las elecciones presidenciales de 2019 y 2018, respectivamente, implicaron alternancias en el poder; no así en el primero. Estos tres países tienen en común el contar con una sola institución electoral, que realiza funciones tanto administrativas como jurisdiccionales. Sin embargo, no puede pensarse que esto sea la causa 
de tan bajos niveles de confianza, ya que por ejemplo Uruguay, que junto a Ecuador representan los dos países latinoamericanos con mayor confianza política en el periodo estudiado, también cuenta con solo un organismo para atender las dos cuestiones (Garcia, 2016). Ambos países, por cierto, tuvieron continuidad en sus gobiernos, el primero por sus resultados electorales de 2015, y el segundo por los registrados en 2017.

Otra conclusión importante del estudio es que confirma algo ya identificado en investigaciones previas, y es el hecho de que, por lo menos en el contexto latinoamericano, la confianza hacia la institución electoral es un fenómeno distinto al de la confianza depositada en otras instituciones de la democracia (Pérez-Verduzco, 2018). Este hallazgo indica la necesidad de que la confianza en las instituciones electorales sea analizada con una perspectiva particular, es decir, sin que esta constituya solamente una variable más dentro del estudio de la confianza política. Al parecer, a las autoridades electorales se les concibe como un actor diferenciado de los partidos políticos, los congresos y los gobiernos, lo cual es bastante sano para los regímenes democráticos. Además, tal percepción está en sintonía con el modelo de organización electoral predominante en la región que es el independiente (García, 2016).

De ahí se desprende la importancia de que los ciudadanos latinoamericanos puedan seguir depositando su confianza en la institución electoral, ya que gran parte de la legitimidad y eficacia de la democracia, al menos en su dimensión electoral, depende de la existencia y el adecuado funcionamiento de este organismo. Como bien señala Woldenberg (2010), construir la confianza suficiente para que todos los actores involucrados en una democracia se comprometan a resolver divergencias mediante la vía electoral, requiere de autoridades electorales imparciales, contiendas equitativas, vías institucionales para dirimir conflictos y que los votos se reflejen de la mejor manera posible en escaños.

Finalmente, una de las limitaciones del presente trabajo es que solamente se enfocó en comparar países latinoamericanos. Por ello, sería interesante que futuras investigaciones contrastaran los niveles de confianza hacia las instituciones electorales en países de diversas regiones del mundo, de tal forma que pudiera indagarse sobre los efectos de variables culturales en este fenómeno. Además, es pertinente saber si se mantiene el hecho de que América Latina es la región con la menor satisfacción con la democracia en el mundo (Corporación Latinobarómetro, 2015). Otra limitación es que el análisis sobre la confianza hacia la autoridad electoral mexicana se ha realizado en un bloque temporal que incluía un único sexenio, es decir, durante un lapso en el que gobernaba el mismo partido (PRI), motivo por el cual valdría la pena que una línea de investigación futura se dirigiera a contrastar los niveles de confianza en la institución electoral aquí analizados con los existentes en el sexenio de Andrés Manuel López Obrador (MORENA).

\section{Referencias}

Alarcón, V. (2016). Reformas político-electorales 20122014 y régimen de gobierno. México: Tribunal Electoral del Poder Judicial de la Federación.

Ayala, J. J., y Triana, J. L. (2020). Reforma electoral de 2014 y calidad de la democracia subnacional en México. Revista Mexicana de Estudios Electorales, 4(23), 47-73.

Barrientos, F. (2010). Confianza en las elecciones y el rol de los Organismos Electorales en América Latina. Revista de Derecho Electoral (10), 1-31.

Corona, A. (2002). Crisis política de 1938 y nuevo rumbo de la Revolución. Contribuciones desde Coatepec, (3), 88-102.

Corporación Latinobarómetro. (2015). La confianza en América Latina 1995-2015. Santiago: Corporación Latinobarómetro.

Denters, B., Gabriel, O., y Torcal, M. (2007). Political confidence in representative democracy. Sociocultural vs. political explanations. En J. W. Van Deth, J. R. Montero, y A. Westholm (Eds.), Citizenship and involvement in European Democracies: a comparative analysis (pp. 6687). Londres: Routledge.

Galindo, M. P. (1986). Una alternativa de representación simultánea: HJ-Biplot. Questiio, 10(1), 13-23.

García, C. (2016). La confianza en las instituciones electorales en las democracias contemporáneas. México en perspectiva comparada. Pluralidad y Consenso, 6(27), 32-65.

Heras, L. L. (2011). Confianza en las instituciones electorales en México: El IFE bajo la mirada ciudadana. Debates, 5(1), 9-23. DOI: https://doi. org/10.22456/1982-5269.20374

Inglehart, R., \& Norris, P. (2017). Trump and the Populist Authoritarian Parties: The Silent Revolution in Reverse. Perspectives on Politics, 15(2), 443454. DOI: https://doi.org/10.1017/S1537592717000111 
Institute for Comparative Survey Research. (2014). World Values Survey. Recuperado el 4 de marzo de 2020, de https://www.worldvaluessurvey.org/WVSOnline.jsp

Instituto Nacional Electoral y El Colegio de México. (2014). Informe País sobre la calidad de la ciudadanía en México. Resumen ejecutivo. México, DF: Instituto Nacional Electoral.

Juárez, I. L. M. (2019). Confianza Institucional en el Instituto Nacional Electoral: un análisis sistémico en el marco de las elecciones federales de 2018 en México [Tesis de maestría]. Instituto de Ciencias de Gobierno y Desarrollo Estratégico. Benemérita Universidad Autónoma de Puebla, Puebla, México.

Kampen, J., Van de Walle, S., y Bouckaert, G. (2006). Assessing the Relation Between Satisfaction with Public Service Delivery and Trust in Government: The Impact of the Predisposition of Citizens Toward Government on Evaluations of Its Performance. Public Performance and Management Review, 29(4), 387-404. DOI: https://doi.org/10.1080/15309576.2006.11051881

Landau, M. (2009). La conflictiva relación entre participación institucionalizada y confianza: El caso de buenos aires. Perfiles Latinoamericanos, 17(34), 111124.

Lin, G., y Chen, L. (2006). Identification of homogeneous regions for regional frequency analysis using the selforganizing map. Journal of Hydrology, 324(14), 1-9. DOI: https://doi.org/https://doi.org/10.1016/j. jhydrol.2005.09.009

Méndez, I., y Loza, N. (2013). Instituciones electorales, opinión pública y poderes políticos locales en México. En Revisión (Vol. 171). Ciudad de México: FLACSO MÉXICO.

Monsiváis, A. (2019). La calidad percibida de los servicios públicos locales y la confianza institucional en México. Región y Sociedad, 31, e1206. DOI: https://doi.org/10.22198/rys2019/31/1206

Montero, J. R., Zmerli, S., y Newton, K. (2008). Social trust, political confidence, and satisfaction with democracy. Reis: Revista española de investigaciones sociológicas, (122), 11-54. DOI: https://doi.org/10.2307/40184879

Moreno, A. (2003). El votante mexicano. México D.F.: Fondo de Cultura Económica.

Ortega, R. Y., y Somuano, M. F. (2014). Estudio sobre la confianza en el Instituto Federal Electoral. Ciudad de México: Instituto Nacional Electoral.

Palazuelos, I. (2012). La desconfianza en los partidos políticos y la percepción ciudadana de desempeño gubernamental: México ante América Latina. Revista Mexicana de Análisis Político y Administración Pública, 1(1), 79-107.

Pérez-Verduzco, G. (2018). Confianza política en
Latinoamérica. Un análisis a través del HJ-Biplot. $56^{\circ}$ Congreso Internacional de Americanistas (pp. 483-497). Salamanca: Universidad de Salamanca.

Segovia, C., Haye, A., González, R., Manzi, J., y Carvacho, H. (2008). Confianza en instituciones políticas en Chile: un modelo de los componentes centrales de juicios de confianza. Revista de ciencia política (Santiago), 28(2), 39-60. DOI: https://doi.org/10.4067/S0718-090X2008000200002

Temkin, B., Salazar, R., y Ramírez, G. (2004). Explorando la dinámica del "Abstencionismo ilustrado": ¿un caso de demasiada o poca cultura democrática? México, D.F.: Facultad Latinoamericana de Ciencias Sociales (FLACSO).

Torres-Ruiz, R. (2014). México y su nueva reforma político electoral. Revista mexicana de estudios electorales, 14, 119-159.

Torrico, M. A. (2015). Gobernanza electoral y confianza ciudadana en el Estado de México. Espiral. Estudios sobre Estado y Sociedad, 22(62), 101-138. https://doi.org/10.32870/espiral.v22i62.249

Valdez, A., \& Huerta, D. A. (2018). El financiamiento de los partidos políticos y la confianza ciudadana en México. Intersticios sociales (15), 309-331.

Valles, R. M. (2016). Elecciones presidenciales 2006 en México. La perspectiva de la prensa escrita. Revista Mexicana de Opinión Pública, 20, 3151. https://doi.org/10.1016/j.rmop.2015.12.003

Woldenberg, J. (2010). La ciudadanización del IFE. En Instituto Federal Electoral. 20 años (pp. 156177). México: Instituto Federal Electoral. 